\title{
El adverbio vũv como marcador discursivo en el Corpus Lysiacum
}

The adverb vũv as a discourse marker in the Corpus Lysiacum

Raquel Fornieles Sánchez ${ }^{*}$

Universidad Autónoma de Madrid, España

raquel.fornieles@uam.es

\section{Resumen:}

Presentamos un estudio del adverbio vข̃v (y su variante reforzada vขví) en el Corpus Lysiacum, con el propósito de describir su funcionamiento como marcador discursivo en contraste con su uso como adverbio prototípico de tiempo.

Palabras Clave: Adverbios de tiempo, vũv, vuví, Marcadores discursivos, Lisias.

\section{Abstract:}

This paper offers a study of the adverb vच̃v (and its reinforced variant vvví) in the Corpus Lysiacum. Our purpose is to provide a description to elucidate its behaviour as a discourse marker in contrast with its use as a prototypical adverb of time.

KEYWORDS: Adverbs of time, vข̃v, vขví, Discourse markers, Lysias.

\section{INTRODUCCIÓN}

Como ocurre en otras lenguas, en griego hay una serie de adverbios temporales ${ }^{1}$ que no funcionan únicamente como adverbios prototípicos en el nivel representativo de la oración, sino que desarrollan usos propios de los marcadores del discurso, ${ }^{2}$ es decir, contribuyen a la organización discursiva, favorecen la cohesión textual y guían las inferencias comunicativas. En este trabajo se estudia el comportamiento de uno de ellos en el Corpus Lysiacum, vข̃v, con el objetivo de aportar una descripción sintáctica, semántica y pragmática que permita dilucidar su funcionamiento como marcador discursivo. Nos centraremos primero en el análisis de su uso como adverbio prototípico de tiempo para contrastarlo, después, con una serie de pasajes en los que presenta un uso que hemos denominado transicional y, por último, con otros en los que actúa plenamente como marcador del discurso. ${ }^{3}$

\section{Usos РRототі́picos}

Desde el punto de vista semántico, vข̃v ("ahora") ${ }^{4}$ indica la noción relacional de "datación” y es un adverbio deíctico que sitúa la acción en el presente o el futuro inmediato del hablante. En Lisias se atestigua también vvví, forma reforzada de origen deíctico. Como cabe esperar, lo más usual es que se combine con predicados verbales en presente de indicativo (1) o de futuro si la acción se ubica en el futuro inmediato del hablante: ${ }^{5}$

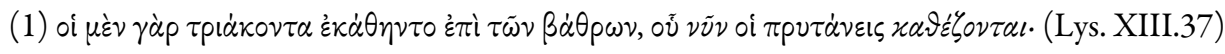

Pues los Treinta se sentaban en los bancos en los que abora se sientan los prítanes. ${ }^{6}$

Con frecuencia, la presencia del adverbio implica una comparación entre una época pasada y el momento presente (2):

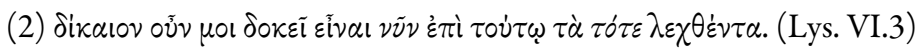

Por tanto, me parece justo recordar ahora, con respecto a este, las cosas que entonces se dijeron. 
De modo mucho menos habitual, alude a un estado de cosas ubicado en el pasado reciente del hablante. En estos casos se combina con formas de aoristo y su significado temporal se vincula a su significado deíctico:

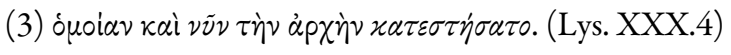

También ahora acaba de establecerse en un cargo semejante.

Estos usos de vĩv con formas verbales de aoristo -documentados ya en los poemas homéricos- ${ }^{7}$ merecen especial atención. En pasajes como el de (3) se describe un estado de cosas situado en un pasado reciente, pero que se extiende hasta el presente del hablante. En estos contextos, vĩv expresa claramente tiempo y funciona como adverbio prototípico. No hay en el Corpus Lysiacum ejemplos en los que el adverbio, en combinación con formas de aoristo, describa estados de cosas que se sitúen en un pasado remoto del hablante. Sin embargo, como veremos, la situación difiere cuando vĩv se comporta como marcador del discurso.

Por otra parte, en los ejemplos que nos ocupan ahora y, a diferencia de lo que ocurre cuando funciona como marcador discursivo, en sus usos como adverbio prototípico admite complementos, puede desempeñar una función sintáctica y suele funcionar como complemento no inherente o adjunto en el sintagma o en el nivel representantivo de la oración. Además, puede estar focalizado, ${ }^{8}$ ser negado ${ }^{9}$ y coordinarse con otros adverbios. ${ }^{10}$ También funciona como predicado de oraciones copulativas ${ }^{11}$ y como modificador del núcleo de un sintagma, como en (4):

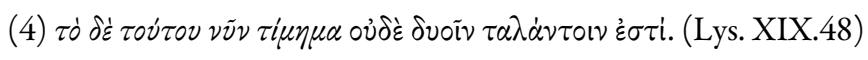

Pero su tasación actual no es ni de dos talentos.

\section{USOS TRANSICIONALES}

El Corpus Lysiacum ofrece pasajes en los que vข̃v presenta un uso transicional. El adverbio expresa nítidamente tiempo pero, a la vez, ejerce una función claramente contrastiva en pasajes de naturaleza argumentativa. En ocasiones, el hablante utiliza el adverbio para ordenar sus ideas y captar la atención del auditorio. ${ }^{12}$ Otras veces, el hablante construye su argumentación contrastando lo ocurrido en una época pasada y lo que sucede en la actualidad, como en (5), donde el orador advierte a los jueces de lo inoportuno de incurrir en una contradicción:

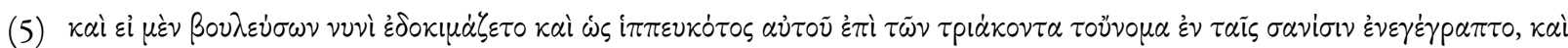

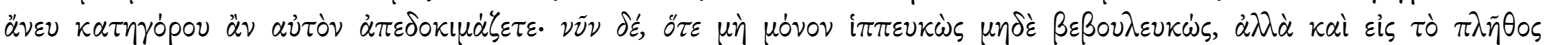

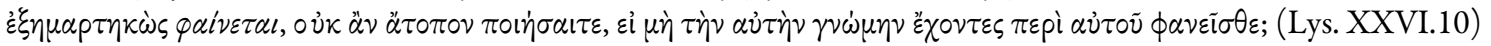

Y si ahora se le examinara para ser miembro del Consejo y, por haber sido caballero en época de los Treinta, su nombre estuviera inscrito en las tablillas, incluso sin acusador lo rechazaríais. Ahora, cuando es evidente que no solo fue caballero y miembro del Consejo, sino que también ha errado contra el pueblo, ¿no actuaríais de modo extraño si no mostrarais la misma opinión sobre él?

En este caso, vข̃v mantiene intacto su significado nocional de tiempo y lo corrobora la presencia de la subordinada temporal que introduce ö $\tau \varepsilon$ a continuación, pues la combinación de $\nu \tilde{\nu}$ con presente -aquí, $\phi \alpha i v \varepsilon \tau \alpha l-e s ~ h a b i t u a l$ en griego para describir una situación en desarrollo en el presente del hablante. ${ }^{13}$

En estos contextos, ขข̃v presenta dos características propias de los marcadores discursivos: se sitúa en posición inicial y siempre se asocia a otro marcador, ${ }^{14}$ en este caso la partícula $\delta \varepsilon$. Sin embargo, su valor temporal aún es nítido, por lo que no puede afirmarse que funcione plenamente como marcador discursivo. Por otro lado, se combina casi en exclusiva con formas verbales de presente o perfecto. Son muy escasos los ejemplos en los que el verbo es un aoristo y, cuando eso ocurre, las situaciones descritas nunca se sitúan en un pasado remoto, sino en un pasado reciente cuyas consecuencias se prologan hasta el presente del hablante, como en (6): 


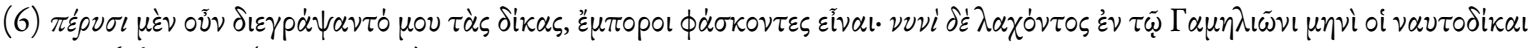
oủ $\dot{\varepsilon}^{\prime} \xi \varepsilon \delta i x \alpha \sigma \alpha \nu$. (Lys. XVII.5)

Pues bien, el año pasado me sobreseyeron la causa afirmando que eran comerciantes. Ahora, en cambio, aunque se me permitió intentar el proceso en el mes de Gamelion, los jueces marítimos no han dictado sentencia.

\section{Usos COMO MARCADOR DISCURSIVO}

Cuando funciona como marcador del discurso, ṽ̃v -siempre asociado a $\delta \varepsilon$ - reúne unos requisitos concretos: no suele ocupar cualquier posición en la oración que lo alberga, sino encabezarla. ${ }^{15}$ Tampoco modifica al predicado, no responde a una interrogación parcial, no puede ser negado, estar focalizado ni coordinarse con otros adverbios; pero, con mucha frecuencia, se combina con otras partículas o conjunciones coordinantes y refuerza su significado.

Por otra parte, muestra un significado procedimental o de procesamiento, ${ }^{16}$ esto es, proporciona instrucciones que guían las inferencias de la comunicación entre el hablante y su interlocutor, permitiendo que esta se lleve a cabo eficazmente. En este sentido vĩv-como el resto de marcadores- funciona en el plano conectivo, pero también puede hacerlo en otros dos planos: el modalizador (poniendo de relieve la modalidad epistémica del mensaje) ${ }^{17}$ y el interactivo (característico en el diálogo, indicando acuerdo o desacuerdo con las palabras del interlocutor o con su actitud, mostrando un deseo de mantener el contacto comunicativo, etc.). Como mostraremos en las siguientes páginas, estos planos no son restrictivos: un mismo marcador discursivo, en este caso $v \tilde{v}$, puede funcionar en varios a la vez.

\subsection{Nĩv en actos de habla directivos, interrogativos y expresivos}

En otros corpora bien estudiados, una de las funciones de vข̃v es expresar un cambio de tópico. Esto sucede en los diálogos y, solo de modo esporádico, en los monólogos como los que nos ocupan. En los pocos contextos en los que vĩv actúa como expresión de cambio de tópico en nuestro corpus, encabeza un miembro discursivo con el que el orador apela a los jueces con dos propósitos: invitarlos a que tomen una decisión o a que escuchen su versión tras haber refutado los cargos que se le imputan: ${ }^{18}$

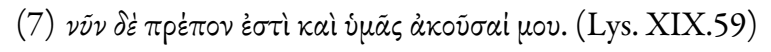

Pero ahora es conveniente que me escuchéis a mí.

Son escasos también en el Corpus Lysiacum los pasajes en los que el adverbio funciona en actos de habla interrogativos o expresivos. Se limitan a algunos contextos en los que el orador plantea una pregunta retórica $\mathrm{o},{ }^{19}$ a otros, en los que el miembro introducido por el adverbio denota la indignación del hablante hacia su adversario:

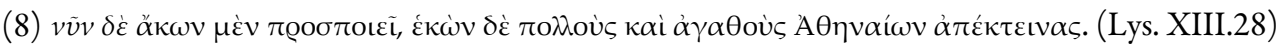

¡Y ahora finges haberlo hecho involuntariamente, aunque hiciste matar voluntariamente a muchos atenienses honrados!

\subsection{Marcador adversativo en actos de habla asertivos}

En un considerable número de ejemplos, vũv funciona como marcador adversativo o contraargumentativo en actos de habla asertivos, uniendo dos miembros discursivos con distinta orientación argumentativa: el segundo -encabezado por el adverbio- se opone a la conclusión a la que conduce el primero. Cuando se comporta así, vข̃v actúa como marcador adversativo de eliminación, ${ }^{20}$ señalando que la información que se ofrece en el segmento que lo alberga reemplaza la información anterior. 
En todos estos contextos, $\nu \tilde{v}$ se combina con la partícula $\delta \varepsilon ́$ y refuerza su significado adversativo. ${ }^{21}$ Opera únicamente en pasajes argumentativos en los que el orador recurre a diversas estrategias argumentativas para construir su defensa o su invectiva. En dichas operaciones discursivas, la secuencia gramaticalizada $\nu \tilde{\nu} \delta \delta ́$ introduce una oración que describe una situación real contrapuesta a otra situación real o irreal. ${ }^{22}$ Lo interesante es examinar la ubicación temporal de las situaciones confrontadas.

\subsubsection{Situación real de presente contrapuesta a una situación irreal de pasado}

En (9) el orador se defiende de la acusación de haber arrancado el tocón de un olivo sagrado. Su estrategia es servirse del razonamiento por reducción al absurdo. ${ }^{23}$ Tras exponer los nulos beneficios que habría obtenido y las graves consecuencias que el robo le habría acarreado, apela a lo ridículo que habría sido correr tal riesgo, pues podría haberlo hecho en cualquiera de sus fincas:

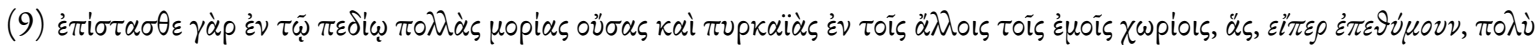

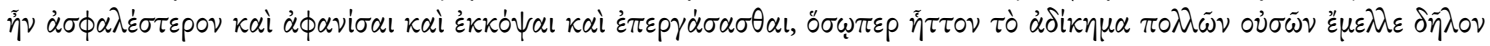

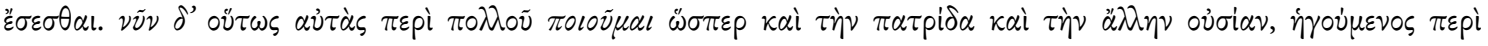

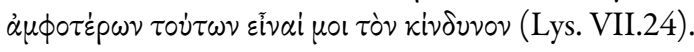

En efecto, sabéis que en la llanura hay muchos olivos sagrados y tocones quemados en mis otros terrenos que, $s i$ realmente lo hubiera deseado, era mucho más seguro haberlo hecho desaparecer, talarlo y cultivar encima en cuanto que, precisamente, al haber un gran número de ellos, el daño iba a ser menos evidente. Ahora bien (Pero el hecho es que), los tengo en tan gran estima como a mi patria y al resto de mi hacienda, porque considero que por ambos corro peligro.

El uso de la conjunción eli $\pi \varepsilon p$ para introducir la situación irreal de pasado en la oración condicional ya es relevante de por sí en la argumentación, pues su uso con imperfecto ( $̇ \pi \varepsilon \theta \dot{v} \mu o v \nu)$ implica que el supuesto que se expone es contrario a la situación que se describe. ${ }^{24}$ La oposición establecida por $v \tilde{v}$ no es temporal, sino que debe entenderse en términos de factualidad: a la situación irreal expresada en el segmento informativo anterior, se contrapone un segmento opuesto introducido por la secuencia $\nu \tilde{\nu} \nu \delta \varepsilon ́$ y marcado expresamente como factual. ${ }^{25}$ En estos casos, lo habitual es que el adverbio se combine con predicados verbales en presente de indicativo.

\subsubsection{Situación real de presente contrapuesta a una situación irreal de presente}

En (10) donde el orador - un inválido acusado de engañar al estado para cobrar una prestación por una invalidez, que, según el demandante, está fingiendo su discapacidad- también argumenta reduciendo al absurdo. Como el procesado indica antes $(\$ 4)$, su rival esgrime como prueba inculpatoria el hecho de que, en su vida cotidiana, monte a caballo, algo que no podría hacer si realmente estuviera incapacitado. Para defenderse, expone que, debido a su escasez de medios, no tiene más remedio que buscar una alternativa:

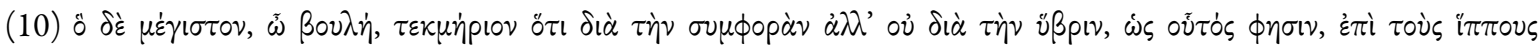

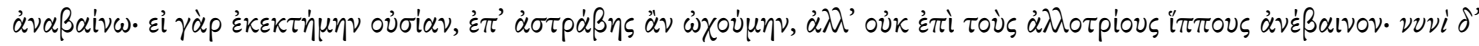

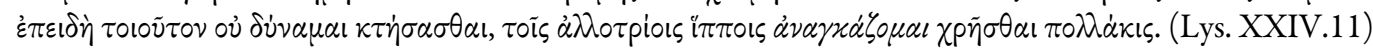

Y esta es la mayor prueba, consejeros, de que es por mi desgracia y no por soberbia, como él dice, por lo que monto a caballo: si tuviera una hacienda, me dejaría llevar en una silla de montar y no me montaría en caballos ajenos. Pero lo cierto es que, puesto que no puedo adquirir tal cosa, me veo obligado a servirme a menudo de los caballos de otros.

Aunque no es simultánea con el momento de la enunciación, la situación descrita se ubica en el presente

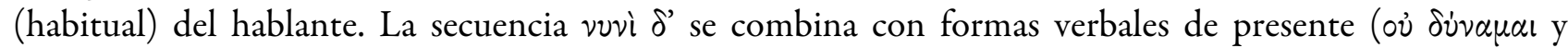
$\dot{\alpha} \nu \alpha \gamma \kappa \dot{\alpha} \zeta o u \alpha \iota)$ que, en este caso concreto, señalan que se trata de una situación frecuente, como remarca la

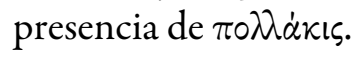


Pese a que la iota incide en la naturaleza deíctica de $\nu \tilde{v} v$ y nos invitaría a pensar únicamente en un funcionamiento como adverbio prototípico, no es posible interpretar un significado nocional claro de vvví como mecanismo para expresar tiempo presente, ya que carece de sentido que el hablante oponga un tiempo presente a otro tiempo presente. Su significado es, por tanto, de procesamiento y el orador lo utiliza como herramienta para contrastar la realidad a la irrealidad. Y es precisamente en la asociación metafórica que se produce entre el tiempo presente y lo que realmente sucede donde se origina el uso de $v \tilde{\nu} \nu$ como mecanismo de expresión de la realidad.

Por otra parte, en todos estos pasajes, el adverbio funciona en el plano conectivo como marcador adversativo de eliminación que refuerza el valor de la partícula $\delta \varepsilon ́$, pero, al mismo tiempo, actúa en el plano modalizador. El adverbio indica el regreso a la realidad y pone de relieve el compromiso asertivo del hablante, que adjudica un alto grado de veracidad a sus palabras. ${ }^{26}$ En este sentido, es interesante prestar atención a algunos aspectos relacionados con la evidencialidad, ${ }^{27}$ como el uso del presente habitual ${ }^{28}$ o el léxico, la

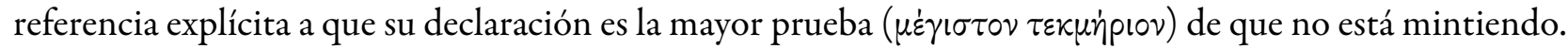

En (11) el procedimiento es el mismo, pero la forma adverbial no es vvví, sino vũv. El acusado trata de demostrar que no ha cometido ningún delito contra la ciudad, sino todo lo contrario: solo le ha proporcionado buenos servicios tanto con su propia persona como con sus bienes y prosigue así su argumentación:

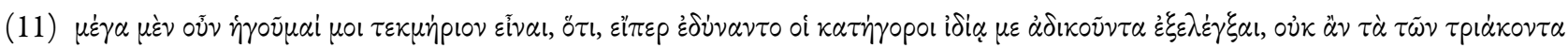

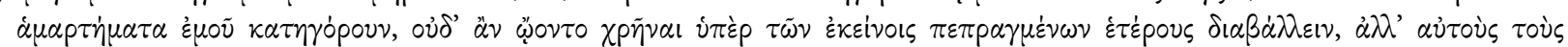

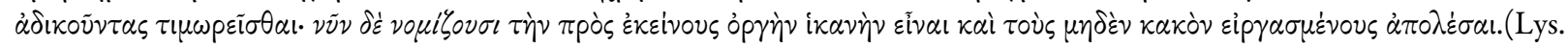
XXV.5)

Pues bien, creo tener una gran prueba en el hecho de que, si realmente pudieran los acusadores demostrar que yo particularmente he delinquido, no me acusarían a mí de las malas acciones de los Treinta, ni considerarían necesario calumniar a otros sobre las acciones llevadas a cabo por estos, sino castigar a los propios culpables. Pero lo cierto es que consideran que su cólera contra aquellos es suficiente incluso para arruinar a quienes no han realizado nada malo.

De nuevo, la secuencia $v \tilde{\nu} \delta \varepsilon ́$ funciona en el plano conectivo y contrapone una situación irreal de presente a

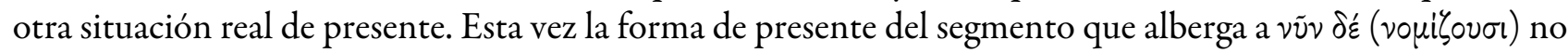
indica que la situación descrita sea habitual, sino que remite a la actualidad, al presente que coincide con el tiempo de la enunciación. Como sucede en el ejemplo anterior, tampoco aquí tiene sentido contraponer una situación de presente a otra situación ubicada en ese mismo presente, por lo que el contraste establecido

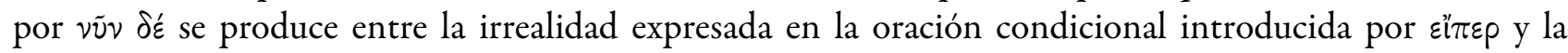
realidad que el acusado refiere para defenderse. ${ }^{29}$

En este sentido, la combinación $\nu \tilde{\nu} \delta$ É actúa nuevamente aquí, al mismo tiempo, en el plano modalizador. También en esta ocasión el acusado se sirve de otras marcas de evidencialidad, como el propio léxico ( $\mu \varepsilon \dot{\gamma} \alpha$ $\tau \varepsilon \kappa \mu \dot{p}(\circ v)$ o el empleo de $\dot{\eta} \gamma o \tilde{u} \mu \alpha \iota$, forma de presente de un verbo de actitud proposicional. ${ }^{30}$

\subsubsection{Situación real de pasado contrapuesta a una situación irreal de pasado}

Los casos que presentamos a continuación son los más interesantes y nos permiten demostrar, sin lugar a dudas, el funcionamiento de $v \tilde{v} v$ como marcador adversativo de eliminación. En (12) el orador, que acaba de aludir a la posibilidad de que los acusadores aleguen que compraron el trigo para beneficiar a la ciudad con el propósito de venderlo más barato, recurre al argumento de los contrarios: ${ }^{31}$ si afirman haber actuado de un modo, no pudieron hacerlo del modo opuesto:

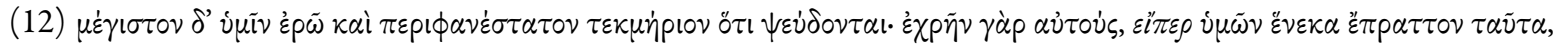

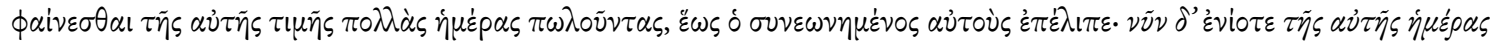

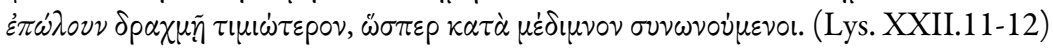


Pero yo os voy a dar la mayor y más visible prueba de que mienten. Pues, si realmente hubieran hecho esto en beneficio vuestro, se les debería haber visto vendiéndolo al mismo precio durante muchos días, hasta que lo que habían adquirido se les hubiera acabado. Pero lo cierto es que algunas veces lo vendían en el mismo día por un dracma más caro, como si lo adquirieran por medimnos.

Las herramientas de las que se sirve el orador son similares a las usadas en (10) y (11). Además de funcionar en el plano modalizador, el adverbio actúa en el plano conectivo pero, a diferencia de los pasajes anteriores, por medio del segmento encabezado por $\nu \tilde{\nu} \delta \delta$, se contrapone una situación real de pasado a otra situación irreal ubicada también en el pasado. El adverbio no expresa tiempo y prueba de ello es que hay otro elemento temporal, $\tau \tilde{\eta} s$ a $\tau \tilde{\eta} s \dot{\eta} \mu \dot{\varepsilon} p \alpha \varsigma$, que hace que la presencia de otro adverbio de tiempo no sea imprescindible. Asimismo, su combinación con una forma verbal de pasado, el imperfecto $\dot{\pi} \pi \dot{\omega} \lambda$ ovv, dificulta la opción de entenderlo literalmente.

Lo mismo sucede en (13), donde el orador ejemplifica con los errores del anterior régimen político ( $\dot{\varepsilon} \nu \tau \tilde{\eta}$

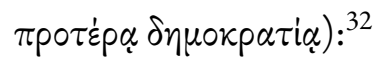

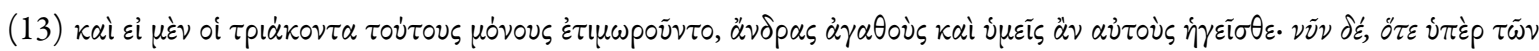

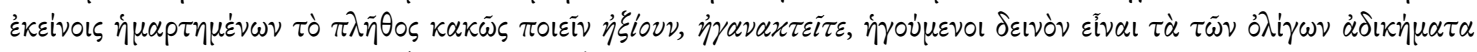

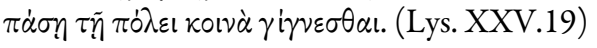

Si los Treinta hubieran castigado solo a estos, incluso vosotros los habríais considerado hombres buenos. Pero lo cierto es que, cuando consideraron conveniente hacer daño al pueblo por los errores cometidos por aquellos, os irritabais, pues creíais que era terrible que las injusticias de unos pocos llegaran a ser comunes a toda la ciudad.

La intención del hablante es disuadir al auditorio de que se cometan los mismos fallos y, para ello, esgrime este argumento que conduce a la siguiente conclusión: "Por tanto, no es justo que os sirváis de los procedimientos con los que los visteis cometer errores". También aquí vĩv $\delta \dot{\varepsilon}$ se combina con una forma verbal de pasado, el imperfecto $\eta \dot{\gamma} \alpha \nu \alpha \kappa \tau \varepsilon i \tau \varepsilon y$, además, antecede a una oración temporal introducida por ö $\tau \varepsilon$ que también sitúa la acción en el pasado ( $\xi_{\xi}$ iovv). A diferencia de lo que sucede en aquellos contextos en los que el adverbio presenta un uso transicional -véase el ejemplo (5), donde la oración de ö $\tau \varepsilon$ tiene el verbo en presente y describe una situación que está en desarrollo en el presente del hablante-, en casos como este la subordinada de ö $\tau \varepsilon$ que sigue a $\nu \tilde{\nu} \delta \dot{\varepsilon}$ anula por completo su significado temporal actual.

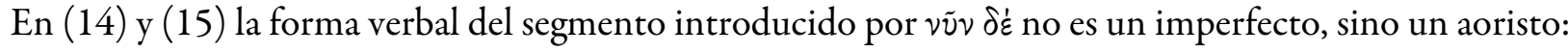

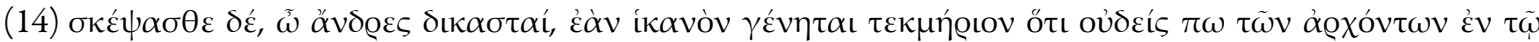

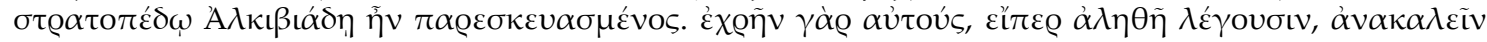

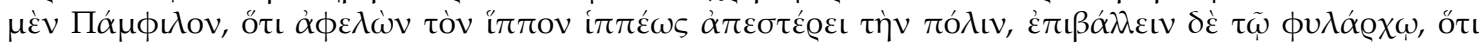

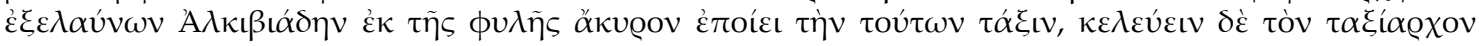

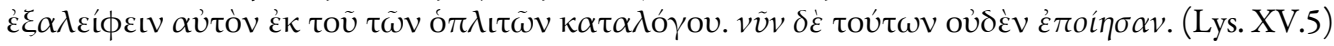

Considerad, jueces, si es suficiente prueba que ninguno de los magistrados del ejército estuviera nunca dispuesto en favor de Alcibíades. Pues, si realmente dicen la verdad, deberían haber hecho comparecer a Pánfilo porque, al quitarle su caballo, privó a la ciudad de un jinete; y haber echado al filarco, porque al expulsar a Alcibíades de su tribu, invalidó el alistamiento; y haber ordenado al taxiarco que lo borrara de la lista de los hoplitas. Pero lo cierto es que no hicieron nada de eso.

Conviene recordar en este punto que en griego tanto el aoristo como el imperfecto pueden referirse alpasado inmediato, reciente o remoto. Sin embargo, la expresión de un pasado remoto es incompatible con el uso de $\nu \tilde{v} v$, un adverbio que ubica la acción en el presente del hablante o en su futuro o pasado inmediatos, pero nunca en su pasado remoto. Lo interesante, por tanto, es observar en qué período de tiempo operan el adverbio y el aoristo. La distinción no es siempre nítida. Sin embargo, en pasajes como el que presentamos en (15) no hay lugar a dudas. El hablante trata de obtener el indulto y se sabe protegido por la ley que permite que un hombre que mata a otro, si lo encuentra cometiendo adulterio, no sea castigado:

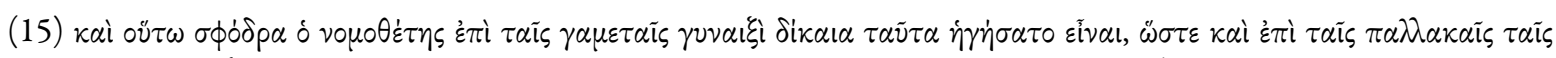

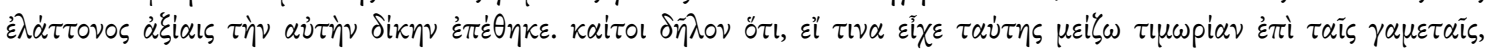

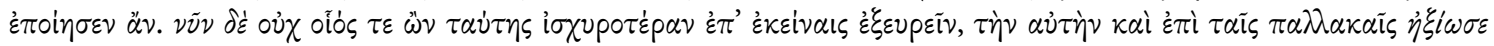

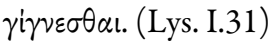


Y tan firmemente creía el legislador que esto era justo en relación con las mujeres casadas, que incluso respecto a las concubinas, menos dignas de estima, impuso la misma pena. Claro que es evidente que si hubiera tenido una pena mayor que esta con respecto a las casadas, la habría impuesto. Pero lo cierto es que, como no le era posible idear un castigo más duro respecto a aquellas, consideró justo que fuera el mismo en relación con las concubinas.

Es obvio que este aoristo no describe una situación ubicada en el pasado inmediato o reciente del hablante, sino en un pasado remoto, pues la ley que menciona el orador fue promulgada por Dracón. ${ }^{33} \mathrm{La}$ combinación de $\nu \tilde{v} \nu \delta \varepsilon ́$ con el aoristo $(\grave{\eta} \xi i \omega \sigma \varepsilon)$ solo se explica si el adverbio funciona plenamente como marcador discursivo, sin significado nocional de tiempo.

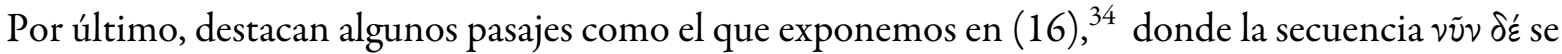
combina con un presente, pero se trata de un presente histórico que introduce, de un modo enfático, la escena del juicio y centra la atención del público en la injusticia cometida contra los demócratas: ${ }^{35}$

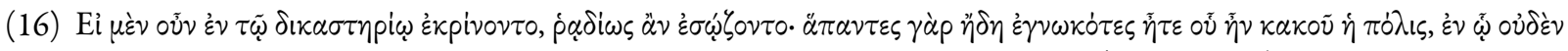

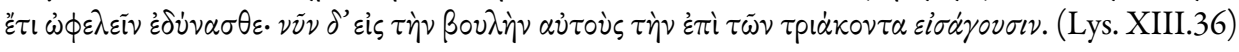

Pues bien, si hubieran sido juzgados en el tribunal, se hubieran salvado fácilmente, pues todos teníais ya conocimiento del mal momento en el que estaba la ciudad, en el que ya no podíais socorrerla en nada. Pero el hecho es que los condujeron ante el Consejo del régimen de los Treinta.

El empleo del presente histórico en casos como este es, por tanto, un recurso estilístico con el que el orador pretende otorgar un efecto dramático a sus palabras y que anula el tiempo transcurrido entre los eventos referidos y su reproducción en la mente de los oyentes con el propósito de que estos se sientan partícipes de lo sucedido como si fueran testigos oculares. ${ }^{36}$ En realidad, la acción se sitúa en el pasado remoto del hablante. No podemos fijar con seguridad cuánto tiempo ha transcurrido entre el momento en el que tiene lugar la situación descrita en el ejemplo y el momento en el que se pronunció el discurso, pero sí que debió ser un período más que considerable, pues el orador pide después, expresamente, a los jueces $(\$ 83)$ que no tengan en cuenta un hecho que Agorato -el acusado- alega en su propia defensa: que el delito ha prescrito.

\section{Conclusiones}

La siguiente tabla muestra la distribución de vũv y vvví en el Corpus Lysiacum:

\begin{tabular}{|c|c|c|c|c|c|c|}
\cline { 2 - 7 } \multicolumn{1}{c|}{} & $\begin{array}{c}\text { Usos } \\
\text { prototípicos }\end{array}$ & $\begin{array}{c}\text { Usos } \\
\text { transicionales }\end{array}$ & $\begin{array}{c}\text { Acto de } \\
\text { habla } \\
\text { directivo }\end{array}$ & $\begin{array}{c}\text { Acto de } \\
\text { habla } \\
\text { interrogativo }\end{array}$ & $\begin{array}{c}\text { Acto de } \\
\text { habla } \\
\text { expresivo }\end{array}$ & $\begin{array}{c}\text { Acto de habla } \\
\text { asertivo: } \\
\text { marcador } \\
\text { adversativo }\end{array}$ \\
\hline $\begin{array}{c}\text { vũv } \\
(130)\end{array}$ & 81 & 16 & 4 & 2 & 2 & 25 \\
\hline $\begin{array}{c}\text { vvví } \\
(50)\end{array}$ & 39 & 8 & - & - & - & 3 \\
\hline
\end{tabular}

Los datos reflejan que tanto vข̃v como la forma reforzada vขví funcionan mayoritariamente como adverbios prototípicos de tiempo en el nivel de la predicación. También se testimonian un notable número de ejemplos 
en los que desempeñan una función claramente contrastiva y presentan usos discursivos, pero conservan intacto su significado nocional de tiempo, lo que indica que se encuentran en un estado transicional.

Cuando se comportan plenamente como marcadores discursivos, el significado de $v \tilde{v} v$ y $v v v i ́$ nunca es temporal, sino de procesamiento y pueden funcionar en los planos conectivo, modalizador e interactivo. Los menos frecuentes son los pasajes en los que vũv (nunca $v v v^{\prime}$ ) opera en actos de habla. Los más relevantes son los contextos en los que vข̃v y vขví, en actos de habla asertivos, son claramente marcadores adversativos de eliminación en pasajes de naturaleza argumentativa, como parte de las diversas estrategias de las que se sirve el orador para construir su defensa o el ataque a su oponente. En el plano modalizador, ponen de relieve la modalidad epistémica del mensaje. En el plano conectivo -se combinan sin excepciones con la partícula $\delta \varepsilon ́$, cuyo carácter adversativo refuerzan- indican que la información que es ofrecida en el segmento en el que se ubican reemplaza la información del segmento anterior.

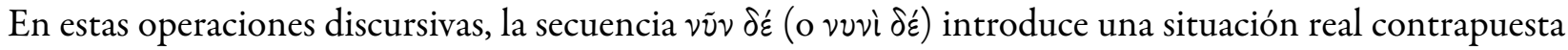
a otra irreal y la ubicación temporal de las situaciones contrastadas es lo realmente interesante. La incompatibilidad del adverbio con formas verbales de pasado o el nulo sentido que tiene el hecho de contraponer un tiempo presente a otro tiempo también presente son las herramientas que mejor nos permiten demostrar que $\nu \tilde{v}$ y $v v v i$ funcionan plenamente como marcadores del discurso. La siguiente tabla refleja el empleo de los adverbios en las distintas variantes:

\begin{tabular}{|c|c|c|c|}
\hline & $\begin{array}{c}\text { Situación real de } \\
\text { presente contrapuesta a } \\
\text { situación irreal de } \\
\text { pasado }\end{array}$ & $\begin{array}{c}\text { Situación real de } \\
\text { presente contrapuesta a } \\
\text { situación irreal de } \\
\text { presente }\end{array}$ & $\begin{array}{c}\text { Situación real de } \\
\text { pasado contrapuesta a } \\
\text { situación irreal de } \\
\text { pasado }\end{array}$ \\
\hline$\nu \tilde{v} v \delta \varepsilon$ & 5 & 7 & 13 \\
\hline $\begin{array}{c}\text { vuví } \\
\text { бé }\end{array}$ & 0 & 2 & 1 \\
\hline
\end{tabular}

\section{REFERENCIAS}

Adams, C. D. (1905) Lysias: Selected Speeches (XII, XVI, XIX, XXII, XXIV, XXV, XXXII, XXXIV), New York.

Aijmer, K. (1988) "Now may we have a word on this. The use of now as a discourse particle", en M. Kytö, O. Ihalainen \& M. Rissanen (eds.) Corpus Linguistics, Hardt and Soft, Amsterdam: 15-34.

Aijmer, K. (2000) English Discourse Particles, Amsterdam.

Aijmer, K. (2013) Understanding Pragmatic Markers, Edinburgh.

Aikhenvald, A. Y. (2004) Evidentiality, Oxford.

Allan, R. J. (2017) “The Grammaticalization of Greek Particles”, en F. Logozzo \& P. Poccetti (eds.) Ancient Greek Linguistics. New Approaches, Insights, Perspectives, Boston: 103-118.

Auer, P. (2016) "Nu(n) in the Upper Saxonian Vernacular of German", en P. Auer \& Y. Maschler (eds.) NU/NA: $A$ Family of Discourse Markers Across the Languages of Europe and Beyond, Freiburg.

Beekes, R. (2010) Etymological Dictionary of Greek, Leiden. 
Blakemore, D. (2002) Relevance and Linguistic Meaning: the Semantics and Pragmatics of Discourse Markers, Cambridge.

Blakemore, D. (2006) “Discourse Markers", en L. R. Horn \& G. Ward (eds.) The Handbook of Pragmatics, Oxford: 221-240.

Brunaud, N. (1991) "Now et then dans la cohérence argumentative", Langages 104: 79-91.

Bybee, J. \& Fleischman, S. (1995) Modality in Grammar and Discourse, Amsterdam.

Calvo Martínez, J. L. (1988) Lisias: Discursos I. Introducciones, traducción y notas, Madrid.

Calvo Martínez, J. L. (1995) Lisias: Discursos II. Introducciones, traducciones y notas, Madrid.

Carey, C. (2000) Lysias: Selected Speeches, Cambridge.

Chantraine, P. (1974) Dictionnaire étymologique de la langue grecque, Paris.

Conti Jiménez, L. (2012) “Zu Konjunktionaladverbien im Altgriechischen: Analyse von ë $\pi \varepsilon \imath \tau \alpha$ bei Homer”, HS 125: 68-80.

Conti Jiménez, L. (2018) “Zur Analyse von vच̃v bei Homer”, IF 123.1: 91-112.

Cornillie, B. (2009) "Evidentiality and Epistemic Modality: On the Close Relationship Between Two Different Categories", Functions of Language 16.1: 44-62.

Crespo Güemes, E., Conti Jiménez, L. y Maquieira Rodríguez, H. (2003) Sintaxis del griego clásico, Madrid.

Crible, L. (2018) Discourse Markers and (Dis)fluency, Amsterdam.

Degand, L., Cornillie, B. \& Pietrandrea, P. (2013) Discourse Markers and Modal Particles, Amsterdam-Philadelphia.

Denniston, J. D. (19542) The Greek Particles, Oxford.

Ducrot, O. (1980) “Analyse de textes et linguistique de l'énonciation”, en O. Ducrot et alii (eds.) Les mots du discours, Paris: 7-56.

Fernández-Galiano, M. (1953) Lisias: Discursos I, Madrid.

Floristán Imízcoz, J. M. (2000) Lisias: Discursos III, Madrid.

Fornieles Sánchez, R. (2014) “Eĩ $\tau \alpha$ y हैँ $\varepsilon \imath \tau \alpha$ en la tragedia griega: de adverbios temporales a marcadores del discurso", Minerva 27: 97-118.

Fraser, B. (1998) “Contrastive Discourse Markers in English", en A. H. Jucker \& Y. Ziv (eds.) Discourse Markers, Amsterdam: 301-326.

Fraser, B. (2006) “Approaches to Discourse Markers”, en K. Fischer (ed.) Approaches to Discourse Particles, Amsterdam: 189-204.

Gernet, L. \& Bizos, M. (1967) Lisias: Discours, Paris.

Gil Fernández, L. (1963) Lisias: Discursos II, Madrid.

Greenbaum, S. (1969) Studies in English Adverbial Usage, London.

Holmes, D. H. (1895) Index Lysiacus, Bonn.

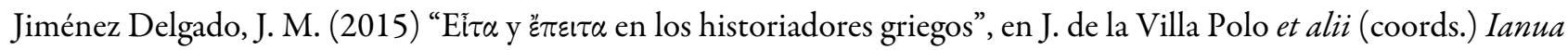
Classicorum: temas y formas del mundo clásico (I), Madrid: 523-530.

Kroon, C. (1998) “A Framework for the Description of Latin Discourse Markers”, J. Pragmat. 30: 205-223.

Lamb, W. R. M. (1930) Lysias, Cambridge.

Lang, E. (2000) “Adversative Connectors on Distinct Levels of Discourse”, en E. Couper-Kuhlen \& B. Kortmann (eds.) Cause - Condition - Concession - Contrast. Cognitive and Discourse Perspectives, Berlin-New York: 235-256.

LSJ= Liddell, H. G. \& Scott, R. (1968) A Greek-English Lexicon, $9^{\text {th }}$ ed. Revised by H. S. Jones, Oxford.

Martín Zorraquino, M. A. y Portolés Lázaro, J. (1999) “Los marcadores del discurso”, en I. Bosque y V. Demonte (eds.) Gramática descriptiva de la lengua española, Madrid: 4051-4207.

Mortier, L. \& Degand, L. (2009) "Adversative Discourse Markers in Contrast. The Need for a Combined Corpus Approach”, International Journal of Corpus Linguistics 14.3: 338-366. 
Murillo Ornat, S. (2010) “Los marcadores del discurso y su semántica”, en Ó. Loureda Lamas y E. Acín Villa (eds.) Los estudios sobre marcadores del discurso en español, hoy, Madrid: 241-280.

Nuyts, J. (2001) Epistemic Modality, Language and Conceptualization: A Cognitive-Pragmatic Perspective, Amsterdam.

Palmer, F. R. (2001) Mood and Modality, Cambridge.

Perelman, Ch. y Olbrechts-Tyteca, L. (1989) Tratado de la argumentación. La nueva Retórica, Madrid.

Portolés Lázaro, J. (1998) Marcadores del discurso, Barcelona.

Quirk, R., Greenbaum, S., Leech, G. \& Svartvik J. (1985) A Comprehensive Grammar of the English Language, LondonNew York.

Ranger, G. (2018) Discourse Markers. An Enunciative Approach, Avignon.

Risselada, R. (1996) "And now for something completely different? Temporal discourse markers: Latin nunc and English now”, en R. Risselada, J. R. de Jong \& A. M. Bolkestein (eds.) On Latin: Linguistic and Literary Studies in Honour of Harm Pinkster, Amsterdam: 105-122.

Rudolph, E. (1996) Contrast. Adversative and Concessive Relations and their Expressions in English, German, Spanish, Portuguese on Sentence and Text Level, Berlin-New York.

Ruiz Yamuza, E. (2014) “El adverbio vच̃v como marcador discursivo”, Emerita LXXXII.1: 1-23.

Saeger, B. (2006) "Evidencialidad y modalidad epistémica en los verbos de actitud proposicional en español", Interlingüistica 17: 268-277.

Schiffrin, D. (1987) Discourse Markers, Cambridge.

Schourup, L. (2011) “The Discourse Marker Now: a Relevance-Theoretic Approach”, J. Pragmat. 43.8: 2110-2129.

Sicking, C. M. J. \& Stork, P. (1997) “The Grammar of the so-called Historical Present in Ancient Greek”, en E. J. Bakker (ed.) Grammar. As Interpretation: Greek Literature in Its Linguistic Contexts, Leiden: 131-215.

Todd, S. C. (2011) A Commentary on Lysias, Speeches 1-11, Oxford.

Van Rooy, R. (2016) “The Relevance of Evidentiality for Ancient Greek: Some Explorative Steps through Plato", Journal of Greek Linguistics 16: 3-46.

Volonaki, E. (1998) A Commentary on Lysias's Speeches 13 and 30, London.

Von Fritz, K. (1949), “The So-Called Historical Present in Early Greek”, Word 5.2: 186-201.

\section{Notas}

* Profesora Ayudante Doctora en la Universidad Autónoma de Madrid. Licenciada en Periodismo y en Filología Clásica, se doctoró en febrero de 2015 en la UAM. Su tesis “La transmisión de noticias en la Literatura Griega Antigua” recibió el premio de la Sociedad Española de Estudios Clásicos a la mejor tesis de Griego de 2015. Cuenta con numerosas publicaciones sobre literatura griega. Entre sus trabajos recientes, destaca el libro Destructoras de hombres y de ciudades. Estudios sobre la 'femme fatale' en la literatura griega -del que ha sido coordinadora-, publicado en 2017 en Liceus. En los últimos años ha orientado su carrera a la lingüística griega, especialmente al estudio de la marcación discursiva en la tragedia y la oratoria (en el marco del Proyecto de Investigación "Marcadores del Discurso en Griego Clásico”, financiado por la DGICYT).

1 Remitimos a Conti Jiménez (2012), Jiménez Delgado (2015) o Fornieles Sánchez (2014).

2 La bibliografía sobre estas unidades lingüísticas es inmensa. Además de los que iremos citando, véanse Aijmer (2000 y 2013), Fraser (2006), Kroon (1998), Blakemore (2006), Degand et alii (2013), Ranger (2018) o Crible (2018).

3 Hemos tenido en cuenta los trabajos de Conti Jiménez (2018) y Ruiz Yamuza (2014) sobre vũv, el de Risselada (1996) acerca de nunc, los de Brunaud (1991), Schiffrin (1987: 228-266), Aijmer (1988) y Schourup (2011) sobre now y el de Auer (2006) sobre nun.

4 Para su etimología, véase Chantraine (1974: 758): "Parenté probable avec *newo- véos". Véase además Beekes (2010: 1025).

5 También con formas de perfecto (cf., por ejemplo, Lys. XIII.37), algo previsible por el propio valor del perfecto, que puede referirse a una situación actual, habitual o general. Con futuro, cf. Lys. XXV.17.

6 Las traducciones son propias. Se han cotejado, además, las traducciones citadas en la bibliografía.

7 Cf. Conti Jiménez (2018: 4). 
8 Manejamos los criterios de Greenbaum (1969), recogidos luego en Quirk et alii (1985: 478-652).

9 Cf. Lys. XII.42.

10 Cf. Lys. X.20.

11 Cf. Lys. XXVII.7.

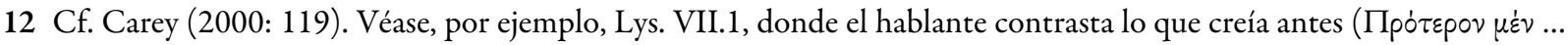

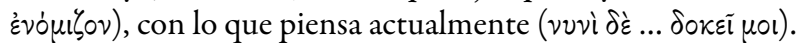

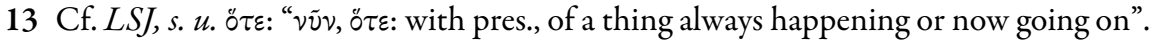

14 A diferencia de otros contextos, como el mostrado en (2).

15 Los marcadores también pueden presentar movilidad, pero siempre que estén acompañados de pausa. Tienden a ocupar posiciones periféricas pero, también, pueden insertarse a modo de cuña en la oración.

16 Ducrot (1980), Portolés Lázaro (1998: 75 y ss.), Martín Zorraquino y Portolés Lázaro (1999: 4071-4080), Blakemore (2002: 89-149) o Murillo Ornat (2010).

17 Sobre el concepto de modalidad, Bybee-Fleischmann (1995), Palmer (2001) o Nuyts (2001).

18 Obsérvese que el hablante no realiza un mandato, sino una invitación (un acto de habla menos coactivo), como muestra

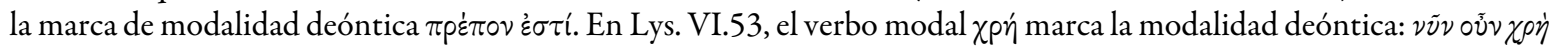

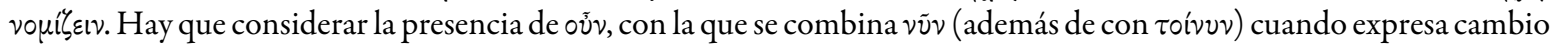
de tópico. En esos casos, la partícula actúa en el plano conectivo, mientras que el adverbio lo hace en el modalizador. Solo en dos contextos el verbo es un imperativo, en Lys. XIII.48 y en Lys. XXVII.

19 Cf. Lys. XII.29.

20 Como los adverbios que Quirk et alii (1985: 635) clasifican como replacive. Los marcadores adversativos presentan diferencias semánticas y se han realizado numerosas clasificaciones. Para una panorámica general, véanse, por ejemplo, Rudolph (1996), Fraser (1998) o Lang (2000).

21 Para este valor de $\delta \dot{\varepsilon}$, véase Denniston $\left(1954^{2}: 165-177\right)$.

22 Sobre la gramaticalización de vข̃v, véanse Allan (2017: 110-115) y Conti Jiménez (2018: 17-18).

23 Cf. Perelman y Olbrechts-Tyteca (1989: 324).

24 LSJ: "In Att. and Trag. to imply that the supposition agrees with the fact, if as is the fact, since, Th.6.14, etc.; but with impf. it implies that it is contrary to the fact, $\iota^{\prime \prime} \pi \varepsilon \rho \tilde{\eta} \nu \pi \dot{\varepsilon} \lambda \alpha \varsigma$ : if I had been (but I was not)". En este caso: "Si realmente lo hubiera deseado (pero no lo deseaba)".

25 Lamb (1930) traduce "But the fact is", marcando precisamente esta idea. Respecto a este tipo de marcadores, véanse, por ejemplo, Mortier \& Degand (2009) o Aijmer (2013: 74-126).

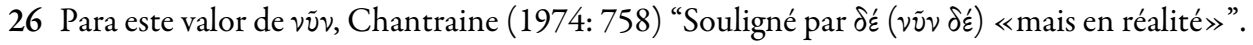

27 De hecho, en uno de los principales estudios sobre la evidencialidad, Aikhenvald (2004: 275) afirma que los marcadores deícticos son importantes fuentes de evidencia: "The source of evidence is established by the speaker, at a specific time and place. Primarily deictic elements may evolve evidentials”. Sobre evidencialidad y modalidad epistémica, también Cornillie (2009). Para el griego, Van Rooy (2016). La evidencialidad es un tema de extrema complejidad que parece tener un enorme rendimiento en la oratoria, por lo que, en adelante, dedicaremos a ella más trabajos.

28 Cf. Aikhenvald (2004: 263).

29 Obsérvese de nuevo el empleo de la conjunción reforzada. Una vez más, el sentido es claro: "Si realmente pudieran los acusadores demostrar que yo particularmente he delinquido (pero no pueden demostrarlo)".

30 Cf. Saeger (2006).

31 Cf. Perelman y Olbrechts-Tyteca (1989: 522-523).

32 Cf. Lys. XXV.18.

33 La ley está recogida en el discurso Contra Aristócrates de Demóstenes. Cf. D. XXIII.53.

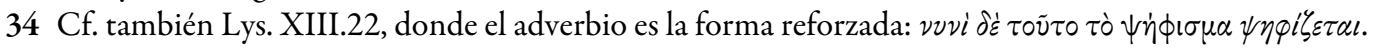

35 Cf. Volonaki (1998: 96).

36 Cf. Von Fritz (1949: 186). Véase también Sicking \& Stork (1997). 